\title{
CONSTRUCTING CHAOTIC TRANSFORMATIONS WITH CLOSED FUNCTIONAL FORMS
}

\author{
WEIHONG HUANG
}

Received 25 May 2006; Accepted 6 June 2006

The properties of complete chaotic unimodal transformations with closed functional forms are examined and the necessary and sufficient conditions are provided to construct such transformations. Theoretical findings are verified with computer simulations.

Copyright (C) 2006 Weihong Huang. This is an open access article distributed under the Creative Commons Attribution License, which permits unrestricted use, distribution, and reproduction in any medium, provided the original work is properly cited.

\section{Introduction}

One of the most unique characteristics demonstrated in chaotic dynamical systems is the stable frequency resulted from long-run trajectories, the so-called invariant density. The study of relationship between the analytical structure of a chaotic transformation and its invariant density has been one of the most difficult tasks. Little progress has been made along the endeavor of deriving the analytical structure of invariant density directly from a chaotic transformation $[4,11]$. Instead, the inverse Frobenius-Perron approach, that is, finding nonlinear chaotic transformations that preserve prescribed invariant densities, dominates the research trend $[2,3,9,10]$. Recently, attempts have been made in deviating this approach by exploring generic "macro" characteristics exhibited in some classes of chaotic transformations that share certain similar analytical properties in the invariant densities preserved $[1,5,6]$ or in the transformation itself $[7,8]$. The current study advances one step further along the latter direction through exploring the class of complete chaotic transformations that are smooth and can be expressed with closed functional forms.

Complete chaotic transformations with closed functional form such as the logistic map (quadratic transformation) and cusp-shaped map have played an important and indispensable role in the historical development of nonlinear dynamic theory. Their simplicity and analytical characteristics help not only in the practical modeling in both natural and social sciences but also in pedagogical illustrations of various dynamical theories. In practical application, to fit a random-like time series (either empirical or experimental)

Hindawi Publishing Corporation

Discrete Dynamics in Nature and Society

Volume 2006, Article ID 42876, Pages 1-16

DOI 10.1155/DDNS/2006/42876 
that exhibits stable frequency in long-run with a deterministic dynamic system, a system with closed functional form is always the first choice, since no matter the parameter estimation, the comparative study of parameters or the bifurcation analysis can all be carried out more efficiently than it would otherwise be taken for a piecewise transformation.

The paper is organized as follows. Section 2 defines the concept SCC transformation. Section 3 then proposes an approach in terms of sufficient conditions to construct smooth chaotic transformations with closed form. The same methodology developed applies to the construction of analytical chaotic transformations. Section 4 is devoted to a more detailed analysis of sufficient conditions.

\section{Complete chaotic transformations with closed forms}

We start with a general description of complete chaotic transformations defined on a unit-interval $I \doteq[0,1]$. The particular choice of unit-interval as domain is not restrictive since any chaotic transformation defined on a closed interval can always be transformed to a transformation defined on the unit-interval through variable substitution (linear topological conjugation).

A chaotic transformation $F: I \rightarrow I$ is said to be unimodal if there exists a participation point (sectioning point) $x_{c} \in I$ such that the transformation $F$ can be expressed as

$$
F(x)=\left(f_{L}(x), f_{R}(x)\right)_{x_{c}}= \begin{cases}f_{L}(x), & 0 \leq x \leq x_{c} \\ f_{R}(x), & x_{c} \leq x \leq 1\end{cases}
$$

where $f_{L}:\left[0, x_{c}\right] \rightarrow[0,1]$ and $f_{R}:\left[x_{c}, 1\right] \rightarrow[0,1]$ are continuous, differentiable except possibly at finite points, monotonically increasing and decreasing, respectively, and onto the unit-interval in the sense that $f_{L}(0)=f_{R}(1)=0$ and $f_{L}\left(x_{c}\right)=f_{R}\left(x_{c}\right)=1$.

A unimodal transformation $F$ defined in (2.1) is said to be complete chaotic (fully chaotic) if it is (i) ergodic with respect to the Lebesgue measure and (ii) chaotic in the probabilistic sense, that is, an absolutely continuous invariant density $\varphi$ is preserved.

A function defined on a real domain is said to possess a closed form (a closed-format, a non-piecewise-defined form) if it is expressed as a composition of elementary functions, their inverses, and smooth functions that are not piecewise-defined. In mathematics, there is no rigourously and precisely defined antonym for "piecewise-defined function" since all functions can be assumed to be piecewisely defined. The widely adopted terminology_ “closed form" - is regarded as a most closed one. However, the vaguely defined terminology "closed form" does not include "absolute function" in general. However, we will abandon such exclusion since as to be illustrated that any function consisting of an absolute term can be recast to an expression without absolute term. For example, $|1-2 x|$ can be rewritten as $\sqrt{(1-2 x)^{2}}$.

A complete chaotic transformation $F(x)=\left(f_{L}(x), f_{R}(x)\right)_{x_{c}}$ is said to have a closed form if (i) $f_{L}$ and $f_{R}$ have a common functional form, that is, $f_{L} \equiv f_{R} \equiv f$, and (ii) $f$ itself has a closed form. 
Typical examples of complete chaotic transformations that appear with closed forms include

(i) the logistic map

$$
Q(x)=4 x(1-x)
$$

which preserves an invariant density $\varphi_{Q}(x)=1 /(\pi \sqrt{x(1-x)})$;

(ii) the cusp-shaped map modeling the intermittency [4]

$$
C(x)=\sqrt{1-|1-2 x|},
$$

which preserves an invariant density $\varphi_{C}(x)=2 x$;

(iii) the tent map

$$
T(x)=1-|1-2 x|
$$

which preserves a uniform invariant density, that is, $\varphi_{T}(x)=1$.

It can be noted immediately that $Q$ has a genuine closed format in the sense that it can never be recast to a piecewise-defined format, while the transformations $C$ and $T$, respectively, defined in (2.3) and (2.4) are piecewise-defined in nature $\left(f_{L} \neq f_{R}\right)$ since they can be further simplified to $C(x)=(\sqrt{2 x}, \sqrt{2(1-x)})_{1 / 2}$ and $T(x)=(2 x, 2(1-x))_{1 / 2}$, respectively.

To distinguish the complete chaotic transformations that have a genuine closed format like $Q$ with the "fakes" like $C$ and $T$, we thus introduce the following definitions formally.

Definition 2.1. A complete chaotic transformation $F=\left(f_{L}, f_{R}\right)$ is referred to as an NSCC transformation if it can be expressed in a closed format in the sense that $f_{L} \equiv f_{R}$. An NCC transformation $F$ is referred to as an SCC transformation if

(i) $f$ cannot be alternatively recast in piecewise-defined format, that is, $F=\left(\tilde{f}_{L}, \tilde{f}_{R}\right)$, with $\tilde{f}_{L} \neq \tilde{f}_{R}$ in functional form,

(ii) $f$ is first-order differentiable at the sectioning point $x_{c}$, that is, $f^{\prime}\left(x_{c}\right)=0$.

It needs to emphasize that it is quite difficult, if not impossible, to tell just from the functional form alone whether a complete chaotic transformation is SCC or not. For example, $T$ can be also recast as with no absolute term at all:

$$
\begin{gathered}
T_{1}(x)=\frac{2}{\pi} \sin ^{-1}(\sin \pi x), \\
T_{2}(x)=1-\sqrt{1-2 x(1-x)-2 x \sqrt{1-x(2-x)}} .
\end{gathered}
$$

In the definition of SCC, only first-order differentiable smoothness at the sectioning point $x_{c}$ is imposed instead of a more strict requirement of "analyticity of $f$," which demands infinitive differentiability for all $x \in(0,1)$. Therefore, an analytical complete chaotic transformation is definitely an SCC transformation. The converse, however, is not necessarily true, as shown in the next example. 
Example 2.2 (analytical transformations and nonanalytical SCC transformations). The following complete chaotic transformations have genuine closed formats and are differentiable at the respective sectioning points:

$$
\begin{gathered}
F_{1}(x)=4 \sqrt{x}(1-\sqrt{x}), \\
F_{2}(x)=4 \sqrt{1-x}(1-\sqrt{1-x}), \\
F_{3}(x)=\sqrt{x(2-x)\left(1-x^{2}\right)}+x(1-x), \\
F_{4}(x)=\frac{1}{2}-\frac{1}{2} \sqrt[3]{1-8 x(1-x)\left(4 x^{2}-6 x+3\right)\left(4 x^{2}-2 x+1\right)} .
\end{gathered}
$$

The absolutely continuous invariant densities preserved by $F_{i}$ 's are

$$
\begin{gathered}
\varphi_{1}(x)=\frac{1}{4 \sqrt{x(1-\sqrt{x})}}, \\
\varphi_{2}(x)=\frac{1}{2 \sqrt{1-x}}, \\
\varphi_{3}(x)=\frac{2}{\pi \sqrt{1-x^{2}}}, \\
\varphi_{4}(x)=\frac{3(2 x-1)^{2}}{\pi \sqrt{x(1-x)\left(4 x^{2}-2 x+1\right)\left(4 x^{2}-6 x+3\right)}},
\end{gathered}
$$

respectively. The graphs of $F_{i}$ and their invariant densities $\varphi_{i}$ for $i=1,2,3,4$ are shown in Figure 2.1. While $F_{1}, F_{2}$, and $F_{3}$ are analytical in $(0,1), F_{4}$ is not because the derivatives do not exist at the two reflectional points in $(0,1)$.

Remark 2.3. It is interesting to see that $F_{1}$ and $F_{2}$ defined in (2.6) have a similar structure as the logistic map. In fact, any complete chaotic transformation with closed form can be expressed in a logistic-map-like format:

$$
f(x)=4 h(x)(1-h(x))
$$

where $h$ is an invariant measure function defined on the unit-interval $I$.

\section{Construction of NCC transformations}

With a general unimodal chaotic transformation $F$ defined by (2.1), for any given point $x \in I$, there exist two preimages: $f_{L}^{-1}(x)$ and $f_{R}^{-1}(x)$, the functional relation between which can be described by an auxiliary function $\bar{\omega}$ that transforms one preimage of $F$ in a segment to a unique counterpart in the other [10]:

$$
\bar{\omega}(x)= \begin{cases}\omega(x)=f_{R}^{-1}\left(f_{L}(x)\right), & x \in\left[0, x_{c}\right), \\ \omega^{-1}(x)=f_{L}^{-1}\left(f_{R}(x)\right), & x \in\left[x_{c}, 1\right] .\end{cases}
$$




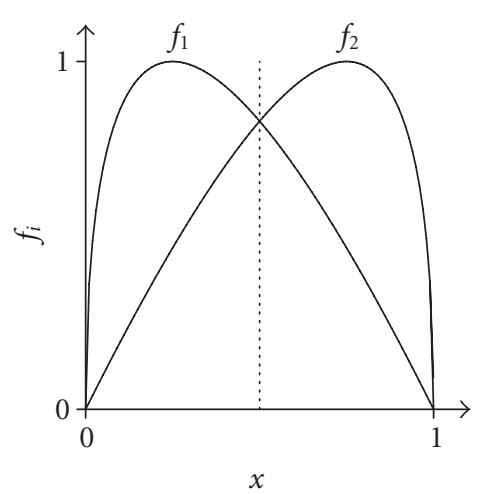

(a)

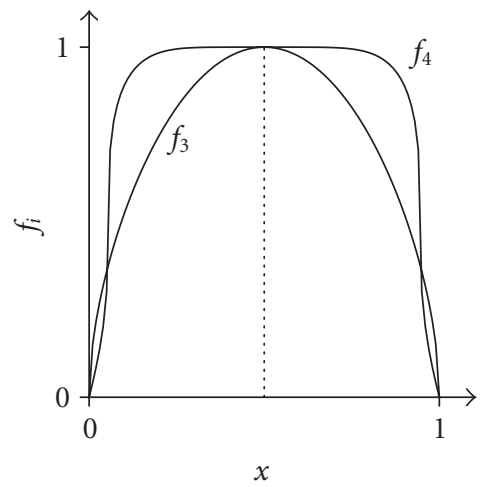

(c)

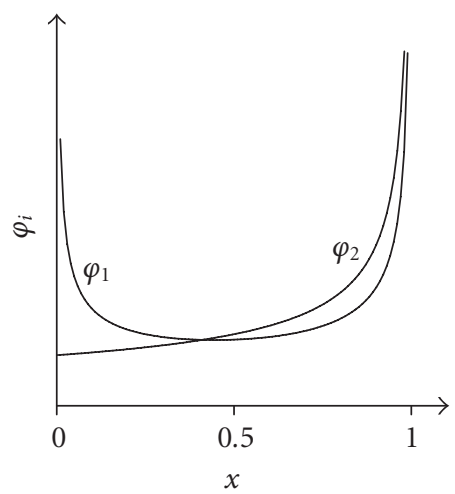

(b)

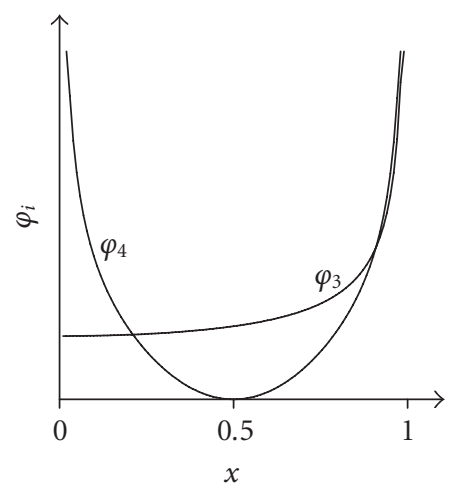

(d)

Figure 2.1. Illustration of SCC maps.

Apparently, $\bar{\omega}$ is differentiable except at finite number of points. Moreover, it is monotonically downward-sloping so that

$$
\begin{gathered}
\omega^{\prime}(x)<0, \quad \text { for almost all } x \in I, \\
\omega(0)=1, \quad \omega\left(x_{c}\right)=x_{c} .
\end{gathered}
$$

For a complete chaotic transformation $F$ preserving an absolute continuous invariant density $\varphi$, denote $\mu(x)=\int_{0}^{x} \varphi(x) d x$ as the related absolute continuous invariant measure, then it is well known that the following Frobenius-Perron equation holds:

$$
\mu(x)=\mu^{-1}\left(F^{-1}([0, x])\right), \quad \forall x \in I,
$$

that is,

$$
\mu(x)=\mu\left(f_{L}^{-1}(x)\right)+1-\mu\left(f_{R}^{-1}(x)\right), \quad \forall x \in[0,1] .
$$


With the auxiliary function $\bar{\omega}: I \rightarrow I$ defined in (3.1), (3.4) can be recast as

$$
\begin{aligned}
F(x) & = \begin{cases}f_{L}(x)=\mu^{-1}(1-\mu(\omega(x))+\mu(x)), & x \in\left[0, x_{c}\right), \\
f_{R}(x)=\mu^{-1}\left(1+\mu\left(\omega^{-1}(x)\right)-\mu(x)\right), & x \in\left[x_{c}, 1\right],\end{cases} \\
& =\mu^{-1}(1-|\mu(\bar{\omega}(x))-\mu(x)|), \quad \forall x \in[0,1] .
\end{aligned}
$$

As piecewise-defined function, $\bar{\omega}$ is not differentiable $x=x_{c}$, neither is $F$ expressed in (3.5).

It can be noticed immediately that as long as $\omega$ has a closed form in $I$ and processes the diagonal-symmetric property in the sense of

$$
\omega(x)=\omega^{-1}(x), \quad x \in I,
$$

the $\bar{\omega}$ function defined in (3.1) will become a closed function so that (3.5) is simplified into

$$
F(x)=\mu^{-1}(1-|\mu(\omega(x))-\mu(x)|), \quad \forall x \in[0,1] .
$$

And hence, as long as neither $\mu$ nor $\mu^{-1}$ is piecewise-defined function, $F$ given by (3.7) is an NCC transformation.

The condition (3.6) will be referred to as the $\omega$-condition. Denote $\Omega$ as the set of $\omega$ functions that meet $\omega$-condition. Typical members of $\Omega$ include

(i) $\omega_{k}(x)=\left(1-x^{k}\right)^{1 / k}, k>0$,

(ii) $\omega_{m}(x)=\left(\left(1-x^{m}\right) /\left(1+x^{m}\right)\right)^{1 / m}, m>0$,

(iii) $\omega_{n}(x)=1-\left(1-(1-x)^{n}\right)^{1 / n}, n>0$,

(iv) $\omega_{\beta}(x)=(1-x) /(1+\beta x), \beta>0$.

Figure 3.2(a) illustrates the several typical members of $\Omega$.

To obtain an SCC transformation, we further need $f_{L} \equiv f_{R}$ and $f_{L}^{\prime}\left(x_{c}\right)=f_{R}^{\prime}\left(x_{c}\right)$, the former identity holds true if and only if

$$
\mu^{-1}(1+\mu(x)-\mu(\omega(x)))=\mu^{-1}(1+\mu(\omega(x))-\mu(x)), \quad x \in[0,1] .
$$

It deserves to warn that although $\mu$ (and hence $\mu^{-1}$ ) is monotonic over its domain $I$, it is not necessarily defined (or keeping the same monotonicity) beyond $I$. And hence, it should not be inferred from (3.8) that $1+\mu(x)-\mu(\omega(x))=1+\mu(\omega(x))-\mu(x)$, which would lead us to a contradictory conclusion: $\omega(x)=x$.

The inequality of $1+\mu(x)-\mu(\omega(x)) \lessgtr 1$ suggests that $1-\mu(x)+\mu(\omega(x)) \gtrless 1$, for $x \in$ I. To meet the condition (3.8), domain of $\mu^{-1}$ should be expanded beyond $I$ and should be symmetric with respect to unity in the sense that

$$
\mu^{-1}(1-x)=\mu^{-1}(1+x), \quad \forall x \in I
$$

Finally, to ensure that $F^{\prime}\left(x_{c}\right)=0, \mu^{-1}$ itself must be differentiable smooth at $x=1$, so is $\omega$ at $x_{c}$. 


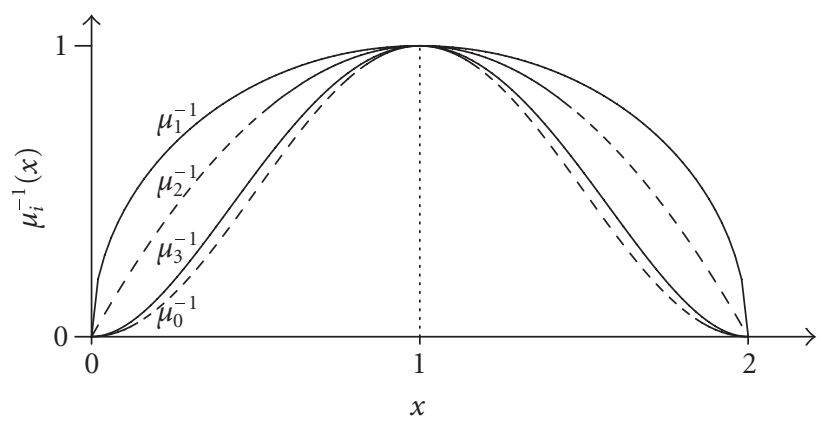

(a) Symmetric and smooth $\mu^{-1}$

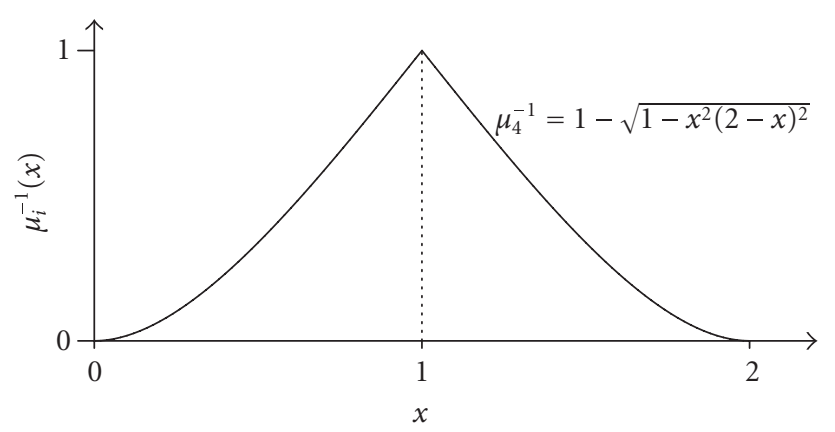

(b) Symmetric but nonsmooth $\mu^{-1}$

Figure 3.1. Odd-symmetricity of $\mu^{-1}$ in $[0,2]$.

The above observations can be summarized into the following proposition.

Proposition 3.1. A unimodal transformation constructed through

$$
f(x)=\mu^{-1}(1+\mu(x)-\mu(\omega(x))), \quad \text { for } x \in[0,1]
$$

is an SCC transformation that preserves the invariant measure $\mu$ if the following conditions are satisfied:

(a) ( $\omega$-condition) $\omega$ has a closed form and is differentiable at $x_{c}$, that is, $\omega^{\prime}\left(x_{c}\right)<0$, and diagonal-symmetric in the sense of (3.6),

(b) ( $\mu$-condition) $\mu^{-1}$ has a closed form and is differentiable at $x=1$, that is, $\mu^{-1 \prime}(1)=0$, and symmetric with respect to the point $x=1$ in the sense of (3.9).

We can check immediately that the invariant measure $\mu_{0}(x)=(2 / \pi) \arcsin (\sqrt{x})$ preserved by the logistic map $Q$ satisfies the $\mu$-condition, and $\omega_{0}(x)=1-x$ implied by the symmetry of $Q$ also meets the $\omega$-condition.

Denote $\mathfrak{M}$ as the set of invariant measures that fulfill the $\mu$-condition. Figure 3.1(a) illustrates $\mu_{0}^{-1}$ along with a few other members of $\mathfrak{M}$ (referring to Example 3.2). Typical 


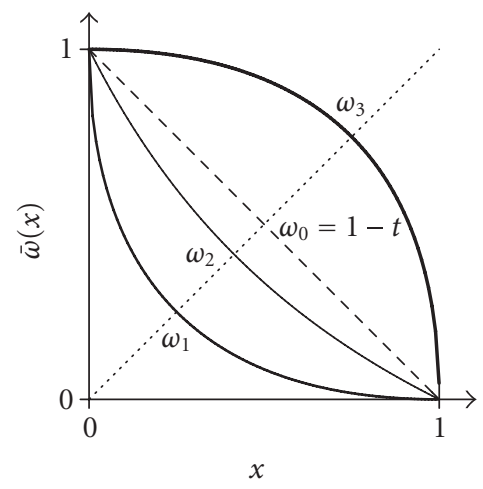

(a) $\omega=\omega^{-1}$

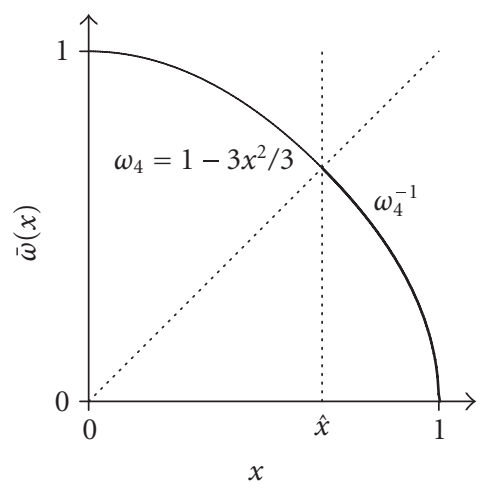

(b) $\omega \neq \omega^{-1}$

Figure 3.2. Diagonal-symmetricity of $\bar{\omega}$ in $[0,1]$.

members of $\mathfrak{M}$ include

(i) $\mu_{\alpha}(x)=(2 / \pi) \sin ^{-1}\left(x^{\alpha}\right), \alpha>0$,

(ii) $\mu_{\beta}(x)=1-(1 / \pi) \cos ^{-1}\left(2 x^{\beta}-1\right), \beta>0$,

(iii) $\mu_{\alpha, k}(x)=1-\left(1-x^{\alpha}\right)^{1 / k}, \alpha>0, k$ is even,

(iv) $\mu_{\beta, m}(x)=(4 / \pi) \arcsin \left(\sqrt{\left(1-\left(1-x^{\beta}\right)^{1 / m}\right) / 2}\right), \beta>0, m$ is even,

(v) $\mu_{\gamma, n}(x)=2 \sin ^{2}(\pi / 4)\left(1-\left(1-x^{\gamma}\right)^{1 / n}\right), \gamma>0, n$ is even.

Before elaborating more on the $\omega$-condition and $\mu$-condition, we offer some SCC transformations that have simple analytical structures. Also provided are the Lyapunov exponents (LCs) that measure the degree of chaoticity of the related SCC transformations. In [8], a simplified formula for calculating LCs is provided:

$$
\Lambda=\int_{0}^{1} \varphi(x) \ln \left(1-\frac{\varphi(\omega(x))}{\varphi(x)} \omega^{\prime}(x)\right) d x
$$

Formula (3.11) enables us to explore LC under different structures of $\omega$ and $\varphi$. For instance, consider the case in which the transformation is symmetric, that is, $\omega(x)=1-x$, we have

$$
\Lambda=\int_{0}^{1} \varphi(x) \ln \left(1+\frac{\varphi(1-x)}{\varphi(x)}\right) d x .
$$

First, we consider the symmetric SCC transformations, which are constructed with $\omega(x)=1-x$.

Example 3.2 (symmetric SCC transformations). With $\omega(x)=1-x$, formulation (3.10) is simplified to

$$
f(x)=\mu^{-1}(1+\mu(x)-\mu(1-x)), \quad \text { for } x \in[0,1] .
$$

Table 3.1 lists some SCC transformations derived from (3.13) along with Lyapunov values resulted from different invariant densities. 
Table 3.1

\begin{tabular}{c|cccc}
\hline$i$ & $\eta_{i}$ & $\mu_{i}^{-1}(x)$ & $\varphi(x)$ & LC \\
\hline 1 & $\sqrt{2} \sqrt{\sqrt{1-x^{2}} \sqrt{2 x-x^{2}}-x(1-x)}$ & $\sqrt{x(2-x)}$ & $\frac{x}{\sqrt{1-x^{2}}}$ & 0.2966 \\
2 & $2 \sqrt{x(1-x)}$ & $x(2-x)$ & $\frac{1}{2 \sqrt{1-x}}$ & 0.5533 \\
3 & $\left(1-(\sqrt{1-\sqrt{x}}-\sqrt{1-\sqrt{1-x}})^{2}\right)^{2}$ & $x^{2}(2-x)^{2}$ & $\frac{1}{4 \sqrt{x(1-\sqrt{x})}}$ & 0.6885 \\
\hline
\end{tabular}

Table 3.2

\begin{tabular}{l|ccc}
\hline$i$ & $\theta_{i}$ & $\omega(x)$ & LC \\
\hline 1 & $(\sqrt{(1-x)(2 \sqrt{x}-x)}+\sqrt{x}-x)^{2}$ & $(1-\sqrt{x})^{2}$ & 0.5927 \\
2 & $\left(4 \sqrt{x\left(1-x^{2}\right)}-x^{2}+5 x+4\right) \frac{x(1-x)}{(x+1)^{2}}$ & $\frac{1-x}{1+x}$ & 0.6857 \\
3 & $(\sqrt{1-x}(1-\sqrt{1-x})+\sqrt{x \sqrt{1-x}(2-\sqrt{1-x})})^{2}$ & $\sqrt{1-x}(2-\sqrt{1-x})$ & 0.5927 \\
\hline
\end{tabular}

The shapes of $\mu_{i}^{-1}$ 's are illustrated in Figure 3.1(a). The functional graphs of $\eta_{i}$ 's together with some typical histograms generated from numerical simulations are presented in Figure 3.3. With a same symmetric structure, SCC transformations that are flatter in the middle range and steeper on the two ends are less chaotic in the sense of lower LC values.

Next, we will examine the relationship between the shape of $\omega$ and the related Lyapunov component for a given invariant density.

Example 3.3 (symmetric densities). For the symmetric invariant measure $\mu_{0}(x)=(2 /$ $\pi) \arcsin (\sqrt{x})$ preserved by the logistic map, Table 3.2 summarizes some SCC transformations resulted from different members of $\Omega$ along with their Lyapunov values.

The graphs of $\omega_{i}$ 's are illustrated in Figure 3.2(a). The functional graphs of $\theta_{i}$ 's together with some typical histograms generated from numerical simulations are presented in Figure 3.4. Even with the invariant density, complete chaotic transformations that are skew to one end are less chaotic in the sense of lower LC values.

\section{More on the sufficient conditions}

It is not clear whether the $\omega$-condition and the $\mu$-condition together are the necessary conditions for a unimodal complete chaotic transformation to have a smooth closed form. Nevertheless, each individual condition is indeed a necessary condition if the other is assumed to hold true. In other words, to construct an SCC transformation, either condition is indispensable if the other is satisfied. If we release the $\mu$-condition and impose 

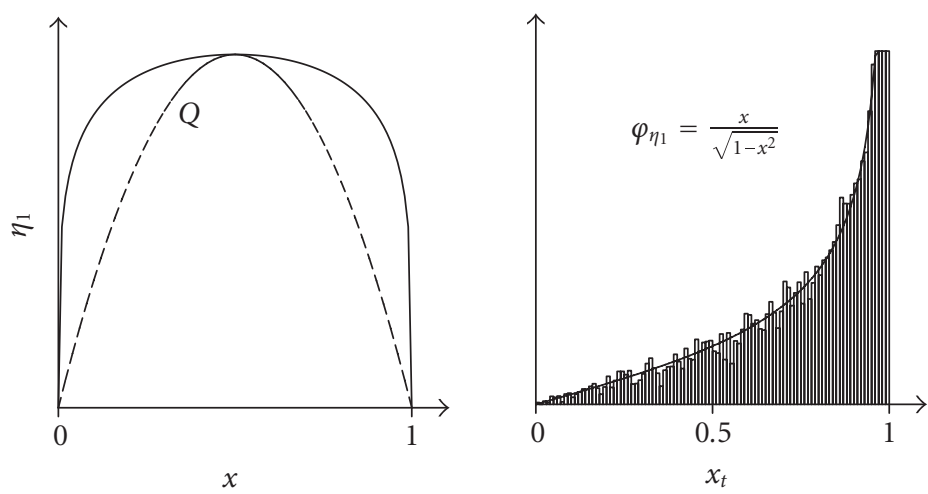

(a) $\eta_{1}(x)=\sqrt{2 x^{2}+2 \sqrt{1-x^{2}}} \sqrt{2 x-x^{2}}-2 x$
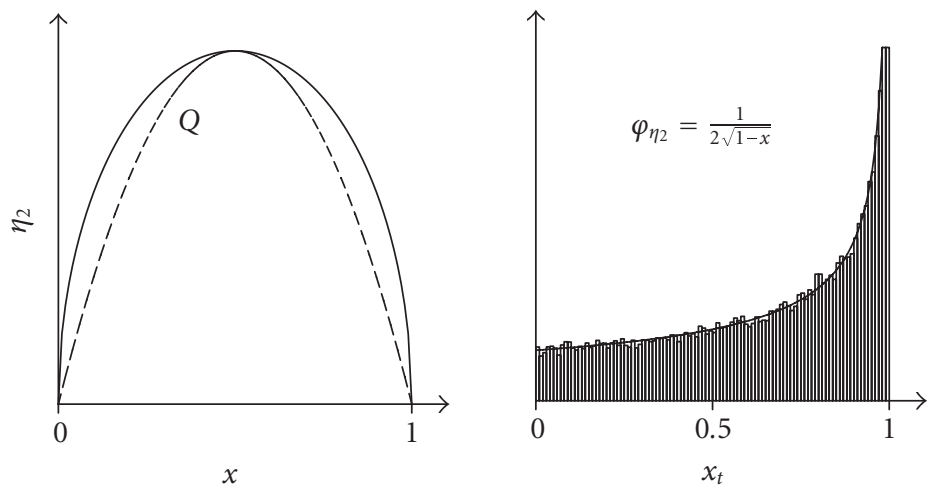

(b) $\eta_{2}(x)=2 \sqrt{x(1-x)}$
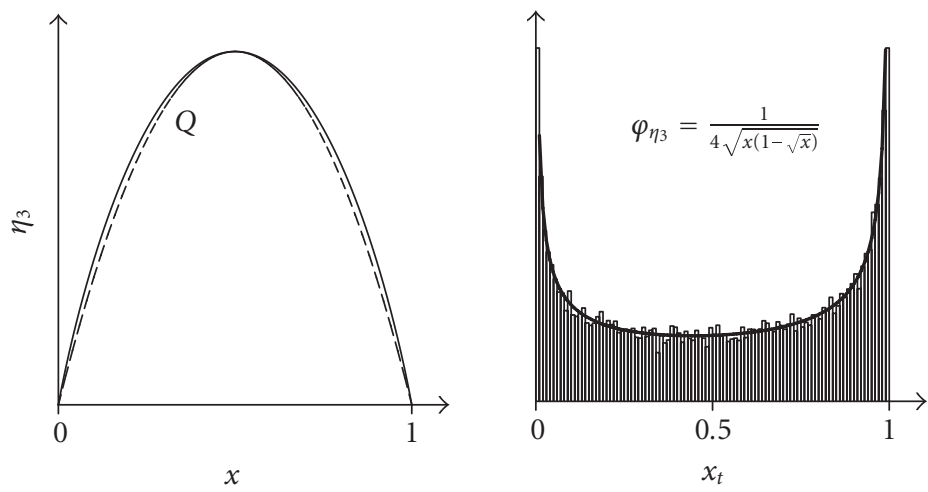

(c) $\eta_{3}(x)=\left(1-(\sqrt{1-\sqrt{x}}-\sqrt{1-\sqrt{1-x}})^{2}\right)^{2}$

Figure 3.3. Symmetric SCC maps preserving different invariant densities. 

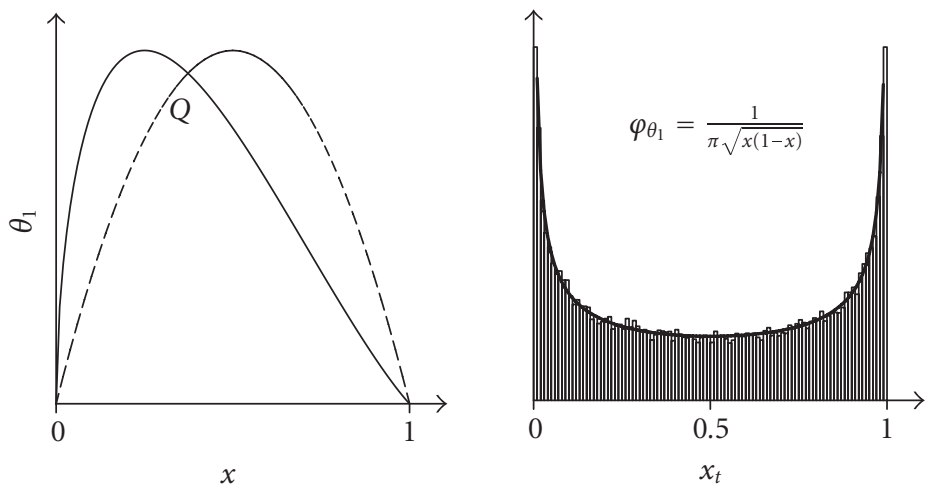

(a) $\theta_{1}(x)=(\sqrt{(1-x)(2 \sqrt{x}-x)}+\sqrt{x}-x)^{2}$
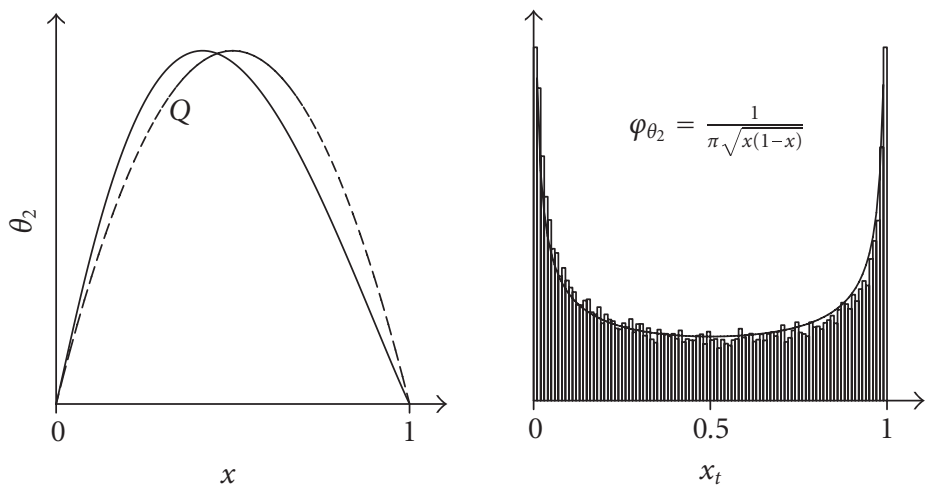

(b) $\theta_{2}(x)=\left(4 \sqrt{x\left(1-x^{2}\right)}-x^{2}+5 x+4\right)\left(x(1-x) /(1+x)^{2}\right)$
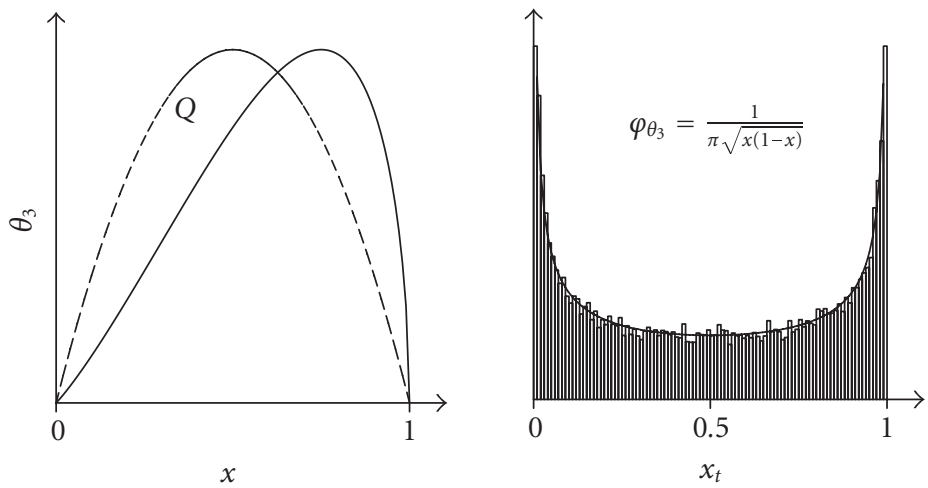

(c) $\theta_{3}(x)=(\sqrt{1-x}(1-\sqrt{1-x})+\sqrt{x \sqrt{1-x}(2-\sqrt{1-x})})^{2}$

Figure 3.4. SCC maps preserving the same symmetric density. 

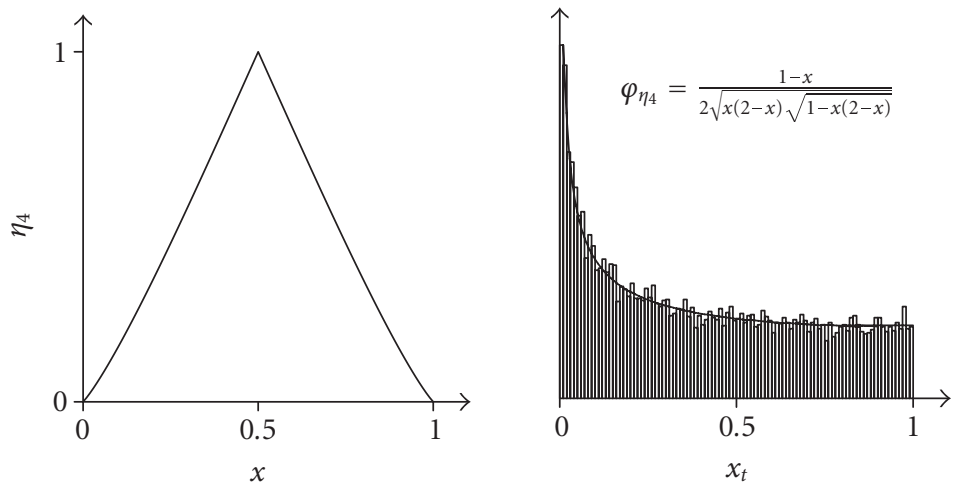

Figure 4.1. Illustration of Example 4.1.

the $\omega$-condition alone, we can only obtain a closed complete chaotic transformation

$$
g(x)=\mu^{-1}(1-|\mu(\omega(x))-\mu(x)|),
$$

which usually but not necessarily contains an absolute value function in its final appearance.

However, it cannot be emphasized more that the "differentiability" and the "closedform" requirements are critical both in the $\omega$-condition and in the $\mu$-condition. The following counterexamples show that how a piecewise-defined $\omega$ function that satisfies (3.6) or a piecewise-defined invariant measure $\mu$ that satisfies (3.9) (even if they are infinitively differentiable elsewhere) results in a nonsmooth complete chaotic transformation.

Example 4.1 (violation of $\mu^{-1}(1)=0$ ). Let $\mu_{4}(x)=1-\sqrt{1-\sqrt{x(2-x)}}$. The inverse of invariant measure is given by $\mu_{4}^{-1}(x)=1-\sqrt{1-x^{2}(2-x)^{2}}$. As illustrated in Figure 3.1(b), although $\mu_{4}^{-1}$ appears with a closed format and is symmetric with respect to $x=1$ as well, it is not differentiable at $x=1$. This is because $\mu_{4}^{-1}$ is essentially a piecewise-defined function over the range $[0,2]$ since it can be recast as $\mu_{4}^{-1}(x)=1-|1-x| \sqrt{1+2 x-x^{2}}$.

If we assume $\omega(x)=1-x$, formulation (3.10) yields a closed but not smooth complete chaotic transformation given by

$$
\begin{aligned}
\eta_{4}(x) & =1-\sqrt{u^{2}(x)\left(2-u^{2}(x)\right)} \\
& =1-|u(x)| \sqrt{2-u^{2}(x)}
\end{aligned}
$$

where $u(x)=\sqrt{1-\sqrt{x(2-x)}}-\sqrt{1-\sqrt{1-x^{2}}}$.

Figure 4.1 shows the graph of $\eta_{4}$ along with a typical realized density from the computer simulations.

The next example shows that a $\omega$ satisfying all requirements except the "closed form" will fail in constructing an SCC transformation. 

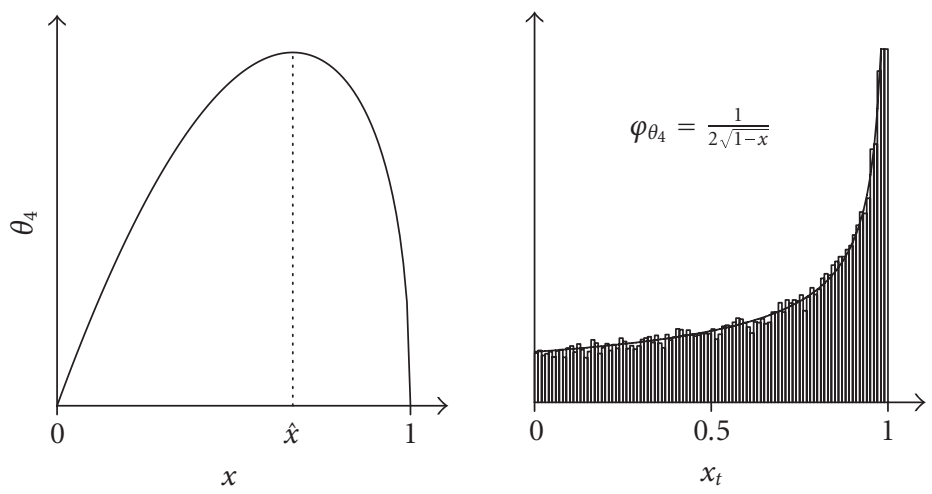

Figure 4.2. Illustration of Example 4.2.

Example 4.2 (violation of $\bar{\omega}=\omega$ ). Let $\mu(x)=1-\sqrt{1-x}$, which satisfies the $\mu$-condition. But if we choose $\omega_{4}(x)=1-(3 / 4) x^{2}$, we have

$$
\bar{\omega}_{4}(x)= \begin{cases}\omega_{4}(x)=1-\frac{3}{4} x^{2}, & 0 \leq x<\frac{2}{3}, \\ \omega_{4}^{-1}(x)=2 \sqrt{\frac{1}{3}(1-x),} & \frac{2}{3}<x \leq 1,\end{cases}
$$

then, as illustrated in Figure 3.2(b), $\bar{\omega}_{4}$ is not only diagonal-symmetric in $[0,1]$ but also smooth and differentiable anywhere in $(0,1)$. Nevertheless, $\bar{\omega}_{4}$ violates the "closed-form" requirement $\left(\omega_{4} \neq \bar{\omega}_{4}\right.$. $)$

It follows from (3.5) that

$$
\begin{aligned}
\theta_{4}(x)= \begin{cases}\mu^{-1}\left(1-\mu\left(\omega_{4}(x)\right)+\mu(x)\right), & 0 \leq x<\frac{2}{3}, \\
\mu^{-1}\left(1+\mu\left(\omega_{4}(x)\right)-\mu(x)\right), & \frac{2}{3}<x \leq 1,\end{cases} \\
= \begin{cases}x\left(1-\frac{3}{4} x+\sqrt{3(1-x)}\right), & 0 \leq x<\frac{2}{3}, \\
x-1+\frac{2}{3} \sqrt{3(1-x)}(1+\sqrt{3-2 \sqrt{3(1-x)}}), & \frac{2}{3}<x \leq 1 .\end{cases}
\end{aligned}
$$

The graph of $\theta_{4}$ is shown in Figure 4.2. It is interesting to see that $\theta_{4}$ is smooth at the sectioning point $x_{c}$ so as to give an illusion of SCC transformation. Also seen in Figure 4.2 is a typical realized density from computer simulations that is consistent with the invariant density $\varphi_{\theta_{4}}(x)=1 /(2 \sqrt{1-x})$.

To form the invariant measures that satisfy $\mu$-condition, the following lemma suggests two constructive approaches. 
Lemмa 4.3. For an analytical two-to-one complete transformation $f$ defined on $I$, if $f$ is symmetric, that is, $f(1-x)=f(x)$, then

$$
\mu(x) \doteq 2 f^{-1}(x)
$$

is a member of $\mathfrak{M}$. On the other hand, if $h$ is an invariant measure function that is oddsymmetric in $I$, that is, $h(x)=1-h(1-x)$, then

$$
\mu(x) \doteq 2 h\left(\frac{1}{2}\left(1-(1-x)^{1 / 2 m}\right)\right)
$$

is a member of $\mathfrak{M}$.

Example 4.4. The logistic map $Q(x)=4 x(1-x)$ and the sinusoidal map $S(x)=\sin (\pi x)$ are two analytical symmetric two-to-one complete transformations defined on $I$. We are able to conclude from (4.5) that $\mu_{1}(x)=2 f^{-1}(x)=1-\sqrt{1-x}$ and $\mu_{2}(x)=2 S^{-1}(x)=(2 /$ $\pi) \sin ^{-1}(x)$ must be two members of $\mathfrak{M}$.

The fact that $h(x)=(2 / \pi) \arcsin (\sqrt{x})$ and $\tilde{h}(x)=\sin ^{2}(\pi / 2) x$ are two odd-symmetric invariant measure functions defined on $I$ brings us two additional members of $\mathfrak{M}$ directly from $(4.6): \mu_{3}(x)=(4 / \pi) \arcsin ((\sqrt{2} / 2) \sqrt{1-\sqrt{1-x}})$ and $\mu_{4}(x)=2 \sin ^{2}(\pi / 4)(1-$ $\sqrt{1-x})$.

The next lemma further sheds lights on the direction of extending and generalizing any member of $\mathfrak{M}$ to more general forms.

Lemma 4.5 (construction rules for $M$ ). (1) Composition rule: let h be an invariant measure function with a closed format, then for any $\mu \in \mathfrak{M}, \hat{\mu} \doteq \mu \circ h \in \mathfrak{M}$ if and only if $h^{\prime}(1) \neq 0$.

(2) Averaging inverse rule: for $\mu_{i} \in \mathfrak{M}, i=1,2$, let $\mu^{-1}=\alpha \mu_{1}^{-1}+(1-\alpha) \mu_{2}^{-1}$, for all $\alpha \in$ $[0,1]$, then $\mu \in \mathfrak{M}$.

(3) Multiplicative inverse rule: for $\mu_{i} \in \mathfrak{M}, i=1,2$, let $\mu^{-1}=\left(\mu_{1}^{-1}\right)^{k}\left(\mu_{2}^{-1}\right)^{l}$, where $k, l>0$, then $\mu \in \mathfrak{M}$. As long as $\lim _{x \rightarrow 0}\left(\mu_{1}^{-1}(x)\right)^{k}\left(\mu_{2}^{-1}(x)\right)^{l}=0$ can be guaranteed, the requirement for strictly positive $k$ and $l$ can be weakened to $k+l>0$.

Proof. (1) Since $\mu \in \mathfrak{M}$, we have $\mu^{-1}(2-x)=\mu^{-1}(x)$ and $\mu^{-1 \prime}(1)=0$. By definition, we have

$$
\begin{gathered}
\hat{\mu}^{-1}(x)=h^{-1}\left(\mu^{-1}(x)\right), \\
\hat{\mu}^{-1}(2-x)=h^{-1}\left(\mu^{-1}(2-x)\right)=h^{-1}\left(\mu^{-1}(x)\right)=\hat{\mu}^{-1}(x) .
\end{gathered}
$$

Since

$$
\hat{\mu}^{-1^{\prime}}(x)=h^{-1 \prime}\left(\mu^{-1}(x)\right) \mu^{-1^{\prime}}(x)
$$

we have

$$
\begin{aligned}
\hat{\mu}^{-1^{\prime}}(1) & =h^{-1^{\prime}}\left(\mu^{-1}(1)\right) \mu^{-1^{\prime}}(1) \\
& =h^{-1^{\prime}}(1) \mu^{-1^{\prime}}(1),
\end{aligned}
$$

which suggests that $\hat{\mu}^{-1^{\prime}}(1)=0$ if and only if $h^{-1^{\prime}}(1) \neq \infty$, or, equivalently, $h^{\prime}(1) \neq 0$.

The proofs for (2) and (3) follow the same principle. 
Remark 4.6. A direct implication of composition rule is that for any $\mu \in \mathfrak{M}$ and $\alpha>0$ we have $\widehat{\mu}(x) \doteq \mu\left(x^{\alpha}\right) \in \mathfrak{M}$.

The requirement for $h^{\prime}(1) \neq 0$ explains why for any $\mu \in \mathfrak{M}$, if we choose $h(x)=\mu^{-1}(x)$, we have $\hat{\mu}=\mu(h(x))=x \notin \mathfrak{M}$. For instance, for the logistic map $Q$, we have $\mu(x)=$ $(2 / \pi) \sin ^{-1}(\sqrt{x})$ so that the choice of $h(x)=\mu^{-1}(x)=\sin ^{2}((\pi / 2) x)$ will lead to $h^{\prime}(1)=0$.

The search for the member of $\Omega$ is more involving and demands more scope. Here, we present a result to be applied later.

Lemma 4.7 (composition rule). Let $h: I \rightarrow I$ be an invariant measure function. For any $\omega \in \Omega\left(x_{c}\right), \widetilde{\omega} \doteq h^{-1} \circ \omega \circ h \in \Omega\left(h^{-1}\left(x_{c}\right)\right)$.

With the above observations, we are able to prove an intuitively true fact.

Proposition 4.8. For any SCC transformation $f$ with a sectioning point $x_{c}$, its topological conjugation defined by $\tilde{f}_{h}=h^{-1} \circ f \circ h$ is also an SCC transformation with a sectioning point $h^{-1}\left(x_{c}\right)$ if and only if $h$ is an invariant measure function that satisfies the differentiability condition $h^{\prime}(1) \neq 0$.

Proof. For $f(x)=\mu^{-1}(1+\mu(x)-\mu(\omega(x)))$, with $\mu \in \mathfrak{M}$ and $\omega \in \Omega\left(x_{c}\right)$, we have

$$
\begin{aligned}
\tilde{f}_{h}(x) & =h^{-1}(f(h(x))) \\
& =h^{-1}\left(\mu^{-1}(1+\mu(h(x))-\mu(\omega(h(x))))\right) \\
& =\tilde{\mu}^{-1}(1+\tilde{\mu}(x)-\tilde{\mu}(\tilde{\omega}(x))),
\end{aligned}
$$

where $\tilde{\mu} \doteq \mu \circ h$ and $\tilde{\omega} \doteq h^{-1} \circ \omega \circ h$. Since $h^{\prime}(x)>0$ for $x \in I$, we conclude from Lemma 4.5 that $\tilde{\mu} \in \mathfrak{M}$ and from Lemma 4.7 that $\tilde{\omega} \in \Omega\left(h^{-1}\left(x_{c}\right)\right)$.

However, $\tilde{f}_{h}^{\prime}(x)=h^{-1 \prime}(f(h(x))) f^{\prime}(h(x)) h^{\prime}(x)$. Denote $\tilde{x}=h^{-1}\left(x_{c}\right)$, then $\tilde{f}_{h}^{\prime}(\tilde{x})=$ $h^{-1 \prime}(1) f^{\prime}\left(x_{c}\right) h^{\prime}(\tilde{x})=0$ if and only if $h^{-1 \prime}(1) \neq \infty$, that is, $h^{\prime}(1) \neq 0$.

Remark 4.9. For an SCC transformation $f$, Proposition 4.8 ensures that $\tilde{f}(x)=\left(f\left(x^{\alpha}\right)\right)^{1 / \alpha}$, $\alpha>0$, is also an SCC transformation. For $\eta_{2}(x)=2 \sqrt{x(1-x)}$ studied in Example 3.2, we immediately know that $f_{1}(x)=\left(\eta_{2}(\sqrt{x})\right)^{2}=4 \sqrt{x}(1-\sqrt{x})$ is also an SCC transformation, which is discussed in Example 2.2. As for the logistic map $Q$, if we choose $h(x)=$ $\cos ^{2} \pi(\sqrt{1-x} / 2)$ so that $h^{-1}(x)=1-\left(4 / \pi^{2}\right) \arccos ^{2} \sqrt{x}$, then we have $h^{\prime}(1)=\pi^{2} / 4 \neq 0$, we thus conclude that

$$
f_{2}(x)=h^{-1}(Q(h(x)))=4 \sqrt{1-x}(1-\sqrt{1-x})
$$

is an SCC transformation, which is shown in Example 2.2.

\section{References}

[1] F. K. Diakonos, D. Pingel, and P. Schmelcher, A stochastic approach to the construction of onedimensional chaotic maps with prescribed statistical properties, Physics Letters. A 264 (1999), no. 2-3, 162-170.

[2] S. Grossmann and S. Thomae, Invariant distributions and stationary correlation functions of onedimensional discrete processes, Zeitschrift für Naturforschung 32a (1977), no. 12, 1353-1363. 


\section{Chaotic transformations with closed forms}

[3] G. Gyögyi and P. Szépfalusy, Complete chaotic 1-d maps Z, Physica B: Condensed Matter 55 (1984), 179-186.

[4] P. C. Hemmer, The exact invariant density for a cusp-shaped return map, Journal of Physics. A: Mathematical and General 17 (1984), no. 5, L247-L249.

[5] W. Huang, Characterizing chaotic processes that generate uniform invariant density, Chaos, Solitons and Fractals 25 (2005), no. 2, 449-460.

[6] __ Constructing an opposite map to a specified chaotic map, Nonlinearity 18 (2005), no. 3, $1375-1391$.

[7] __ Constructing complete chaotic maps with reciprocal structures, Discrete Dynamics in Nature and Society 2005 (2005), no. 3, 357-372.

[8] _ On complete chaotic maps with tent-map-like structures, Chaos, Solitons and Fractals 24 (2005), no. 1, 287-299.

[9] S. Koga, The inverse problem of Frobenius-Perron equations in $1 D$ difference systems. $1 D$ map idealization, Progress of Theoretical Physics 86 (1991), no. 5, 991-1002.

[10] D. Pingel, P. Schmelcher, and F. K. Diakonos, Theory and examples of the inverse Frobenius-Perron problem for complete chaotic maps, Chaos 9 (1999), no. 2, 357-366.

[11] M. Thaler, The invariant densities for maps modeling intermittency, Journal of Statistical Physics 79 (1995), no. 3-4, 739-741.

Weihong Huang: School of Humanities and Social Sciences, Nanyang Technological University,

Nanyang Avenue, Singapore 639798

E-mail address: awhhuang@ntu.edu.sg 


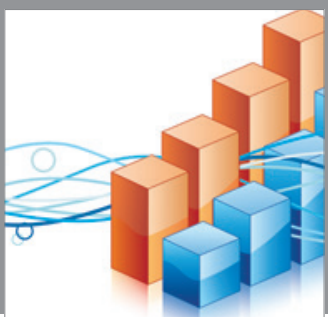

Advances in

Operations Research

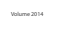

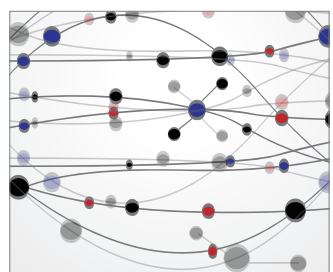

\section{The Scientific} World Journal
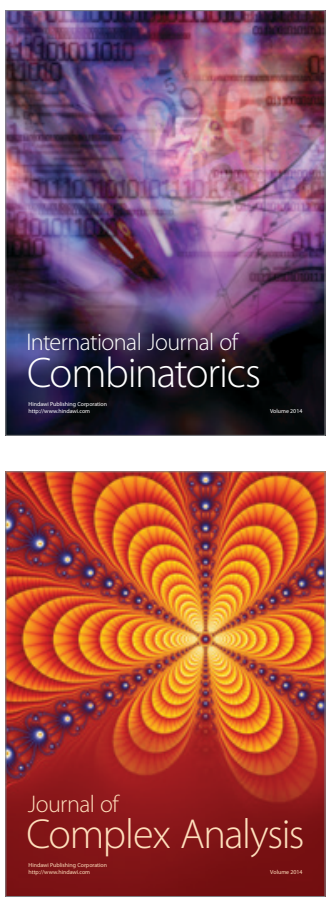

International Journal of

Mathematics and

Mathematical

Sciences
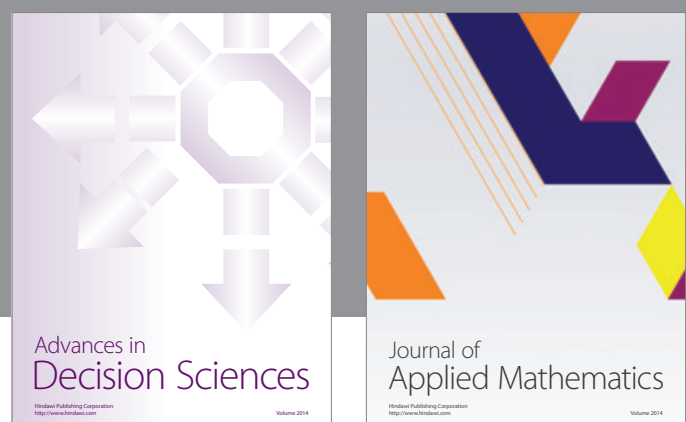

Journal of

Applied Mathematics
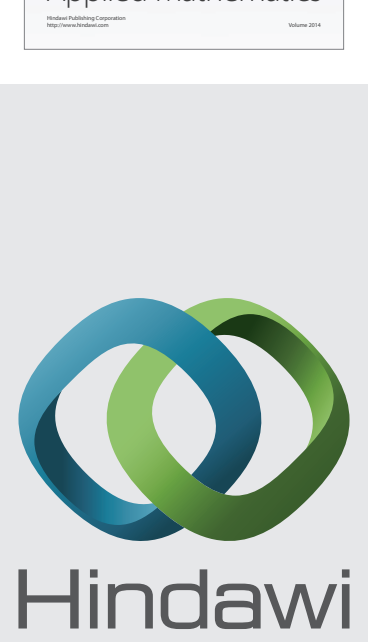

Submit your manuscripts at http://www.hindawi.com
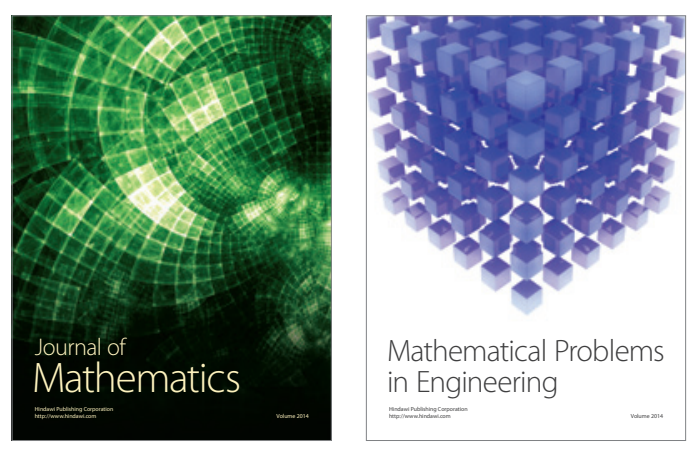

Mathematical Problems in Engineering
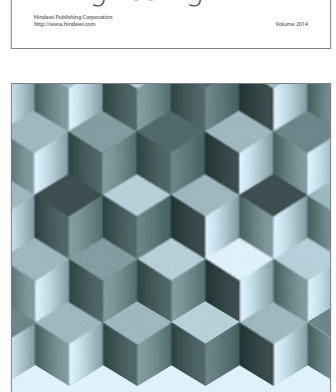

Journal of

Function Spaces
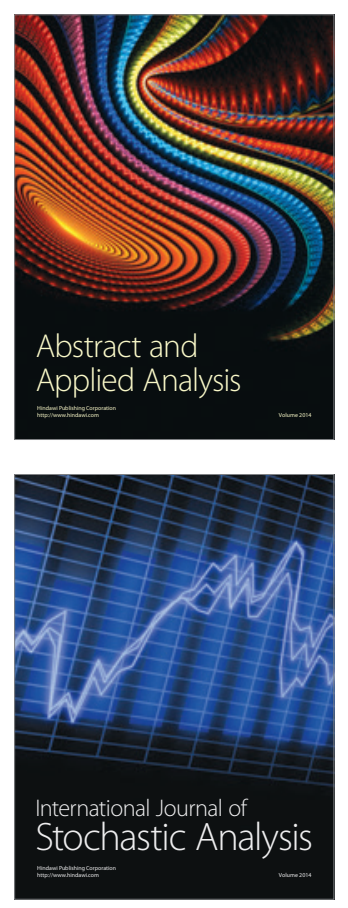

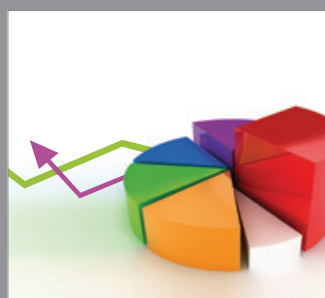

ournal of

Probability and Statistics

Promensencen
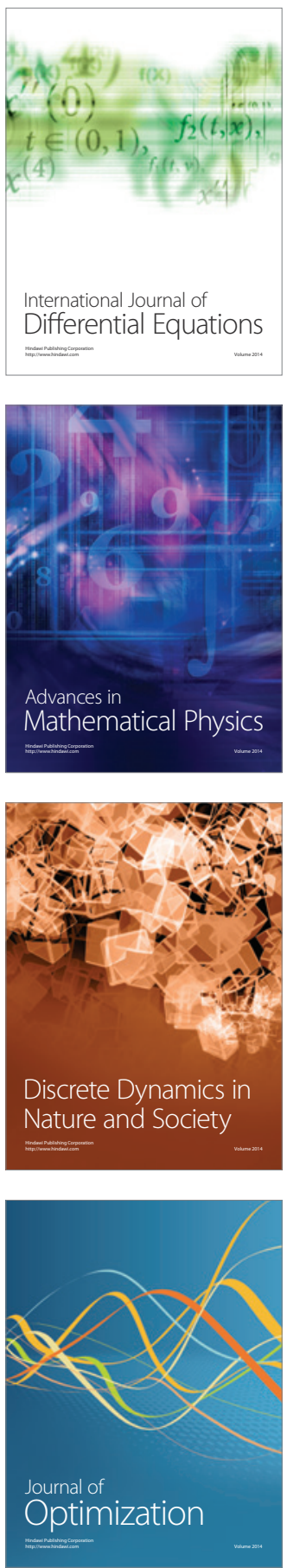